\title{
MYCN and PRC1 cooperatively repress docosahexaenoic acid synthesis in neuroblastoma via ELOVL2
}

Yi Ding ${ }^{1,2+}$, Jie Yang ${ }^{1,2+}$, Yawen Ma ${ }^{1,2}$, Tengteng Yao ${ }^{1,2}$, Xingyu Chen ${ }^{1,2}$, Shengfang G ${ }^{1,2^{*}}$, Lihua Wang ${ }^{1,2^{*}}$ and Xianqun Fan ${ }^{1,2^{*}}$

\begin{abstract}
Background: The MYCN amplification is a defining hallmark of high-risk neuroblastoma. Due to irregular oncogenes orchestration, tumor cells exhibit distinct fatty acid metabolic features from non-tumor cells. However, the function of MYCN in neuroblastoma fatty acid metabolism reprogramming remains unknown.

Methods: Gas Chromatography-Mass Spectrometer (GC-MS) was used to find the potential target fatty acid metabolites of MYCN. Real-time PCR (RT-PCR) and clinical bioinformatics analysis was used to find the related target genes. The function of the identified target gene ELOVL2 on cell growth was detected through CCK-8 assay, Soft agar colony formation assay, flow Cytometry assay and mouse xenograft. Chromatin immunoprecipitation (ChIP) and Immunoprecipitation-Mass Spectrometer (IP-MS) further identified the target gene and the corepressor of MYCN.
\end{abstract}

Results: The fatty acid profile of MYCN-depleted neuroblastoma cells identified docosahexaenoic acid (DHA), an omega-3 polyunsaturated fatty acid with anti-tumor activity, significantly increased after MYCN depletion. Compared with MYCN single-copy neuroblastoma cells, DHA level was significantly lower in MYCN-amplified neuroblastoma cells. RT-PCR and clinical bioinformatics analysis discovered that MYCN interfered DHA accumulation via ELOVL fatty acid elongase 2 (ELOVL2) which is a rate-limiting enzyme of cellular DHA synthesis. Enforced ELOVL2 expression in MYCN-amplified neuroblastoma cells led to decreased cell growth and counteracted the growth-promoting effect of MYCN overexpression both in vitro and vivo. ELOVL2 Knockdown showed the opposite effect in MYCN single-copy neuroblastoma cells. In primary neuroblastoma, high ELOVL2 transcription correlated with favorable clinical tumor biology and patient survival. The mechanism of MYCN-mediated ELOVL2 inhibition contributed to epigenetic regulation. MYCN recruited PRC1 (Polycomb repressive complex 1), catalysed H2AK119ub (histone 2A lysine 119 monoubiquitination) and inhibited subsequent ELOVL2 transcription.

Conclusions: The tumor suppressive properties of DHA and ELOVL2 are repressed by the MYCN and PRC1 jointly, which suggests a new epigenetic mechanism of MYCN-mediated fatty acid regulation and indicates PRC1 inhibition as a potential novel strategy to activate ELOVL2 suppressive functions.

Keywords: Neuroblastoma, Docosahexaenoic acid metabolism, MYCN, ELOVL2

\footnotetext{
* Correspondence: geshengfang@sjtu.edu.cn; wanglihua0902@126.com; fanxq@sjtu.edu.cn

${ }^{+}$Yi Ding and Jie Yang contributed equally to this work.

'Department of Ophthalmology, Ninth People's Hospital, Shanghai JiaoTong

University School of Medicine, Shanghai 200011, China

Full list of author information is available at the end of the article
}

(c) The Author(s). 2019 Open Access This article is distributed under the terms of the Creative Commons Attribution 4.0 International License (http://creativecommons.org/licenses/by/4.0/), which permits unrestricted use, distribution, and reproduction in any medium, provided you give appropriate credit to the original author(s) and the source, provide a link to the Creative Commons license, and indicate if changes were made. The Creative Commons Public Domain Dedication waiver (http://creativecommons.org/publicdomain/zero/1.0/) applies to the data made available in this article, unless otherwise stated. 


\section{Background}

The MYC family of transcription factors, including MYC, MYCN, and MYCL, are the most commonly altered oncogenes in cancer [1, 2]. MYC mediates global metabolic reprogramming to match the enhanced demand for anabolic metabolites in tumor cells [3]. MYC enhances glucose intake and metabolism $[4,5]$, promotes the conversion of glutamine to glutamate and oxaloacetate/pyruvate to aspartate/alanine [6, 7], accelerates fatty acid biosynthesis $[8,9]$. Similarly, recent studies have shown that tumors with an amplified MYCN gene, which is considered the most robust prognostic marker indicating poor outcome for neuroblastoma patients, enhanced glutamine transport and increased glutaminolysis for increased tumor cell growth [10-14]. However, the function of MYCN in global metabolic reprogramming is controversial. Some studies have shown that there is little or no correlation between MYCN amplification and glycolysis $[15,16]$, while no reports so far studied the relationship between MYCN and fatty acid metabolism. MYCN plays a critical role in neuroblastoma tumor initiation, aggressiveness and resistance to chemotherapy [17-19]. Collectively, the potential regulatory role of MYCN in metabolic reprogramming, especially fatty acid metabolism, should be comprehensively investigated to pave the way for novel therapies.

Alterations in fatty acid (FA) metabolism in cancer cells are increasingly being recognized. FAs are the primary source of energy storage, are the components of membrane phospholipids and generate signalling molecules [20]. Of particular interest within the FA family are polyunsaturated fatty acids (PUFAs), because PUFAs metabolism are highly activated in the neuron system [21]. As the main PUFA in neuron phosphatidylserine, DHA (docosahexaenoic acid, C22:6n-3), an omega-3 PUFAs, promotes neuronal survival and neurogenesis in the brain [22, 23]. DHA and its mediator synaptamide increases phosphatidylserine synthesis, and enhances neuronal differentiation, neurite growth, synaptogenesis and synaptic function [24-27]. Furthermore, numerous experimental evidences demonstrate that DHA exerts potent anti-tumor effects on colorectal, breast, bladder, prostate, oesophagus and glioblastoma cancers [28-30]. DHA level in most cancer tissues were found to be lower than those in normal tissues, and associated with a reduced risk of breast, prostate, colon and renal cancers [31, 32].

Interestingly, neuroblastoma initiates from proliferating early neural crest-derived progenitor cells unable to differentiate due to mistakes occurring during embryonal neuroendocrine or sympathoadrenal development [33-35]. Recent evidence also indicated that MYCN is involved in the acquisition and maintenance of pluripotency in neural stem cells [36]. Although there is no reported correlation between MYCN and DHA, the existing evidence suggests that both MYCN and DHA associate with neuroblastoma initiation and development $[37,38]$, and the relationship between these two factors should be comprehensively elucidated to develop novel treatments.

Here, we discovered that MYCN inhibition lead to significant DHA accumulation and upregulated its synthesis enzyme ELOVL2 in neuroblastoma cell lines. We assessed the expression of ELOVL2 in primary neuroblastomas and neuroblastoma cell lines and unravelled its tumor suppressor activity. Furthermore, we elucidated the upstream epigenetic repressive regulation of ELOVL2 through MYCN and PRC1 complex-mediated histone ubiquitination in MYCN-amplified neuroblastoma. The repression of ELOVL2 by the MYCN and PRC1 complex indicates PRC1 complex inhibition as a potential novel strategy to activate ELOVL2 tumor suppressive functions.

\section{Materials and methods \\ Cell culture}

The BE(2)-C, IMR-32 and SK-N-AS cell lines were obtained from the SIBS (Cell Resource Center, Shanghai Academy of Life Sciences, Chinese Academy of Sciences). High-throughput multiplex cell contamination testing was used to monitor cell line infections [39]. High-throughput SNP-based assays were used to validate cell line authenticity [40]. The BE(2)-C, IMR-32 and SK$\mathrm{N}$-AS cell lines were cultured in DMEM (Gibco) supplemented with 10\% FBS (Thermo Fisher Scientific) and 1\% non-essential amino acids (NEAAs; Sigma-Aldrich) at $37^{\circ} \mathrm{C}$ and $5 \% \mathrm{CO} 2$.

\section{siRNAs, plasmids and establishing stable cells}

siRNA oligos targeting BMI1, RING1A, RING1B and the negative control siRNA were purchased from GenePharma and were transfected using Lipofectamine 2000 (Life Technologies) at a concentration of $50 \mathrm{nM}$. All siRNAs used are summarized in Table 1. The two shRNA sequences targeting MYCN and ELOVL2 were cloned into the pGIPZ lentivirus vector (System Biosciences). The CDS sequence of human MYCN, ELOVL2 and SREBP1 were generated by PCR and cloned into the CMV-MCS-EF1-ZsGreen1-PGK-Puro vector (Addgene). Transduction and viral infection were performed as previously described [41].

\section{Lipid extraction and GC-MS}

For lipid extraction, $1 \times 10^{6}$ cells were collected and resuspended in $250 \mu \mathrm{l}$ PBS and $100 \mu \mathrm{l}$ deuterated internal standards. Then, the suspended cell solution was mixed with $500 \mu \mathrm{l}$ methanol and $25 \mu \mathrm{l} 1 \mathrm{~N} \mathrm{HCl}$. A bi-phasic solution was formed by the addition of $1.5 \mathrm{ml}$ of isooctane 
Table 1 Primer, siRNA and shRNA used in this study

\begin{tabular}{|c|c|c|}
\hline Primer & Sequence of forward primer & Sequence of reverse primer \\
\hline MYCN & ACTGTAGCCATCCGAGGACA & TCGGAAGCAGAAACAGTCCC \\
\hline FADS1 & GTTATCCAGCGAAAGAAGTGGG & CCAATAGTGGCACATAAGTGAGG \\
\hline FADS2 & GACCACGGCAAGAACTCAAAG & GAGGGTAGGAATCCAGCCATT \\
\hline ELOVL1 & TTATTCTCCGAAAGAAAGACGGG & ATGACATGCACGGAAGAGTTTAT \\
\hline ELOVL2 & GGAAGCTGACATCCGGGTAG & TCCAGTTCAAGACACACCACC \\
\hline ELOVL5 & CTATTGTCCCGCGATTGGCT & CAGCAGTGTGAGTCCAAGGT \\
\hline ELOVL7 & AGATGCTGATCCAAGAGTTGAAG & GCTGTGGGTGACCGTGAATA \\
\hline SLC27A2 & GTGCTGCACTACTGATTGGC & CCCAGTGCCAGTCTCACTTT \\
\hline SLC27A3 & ACCCTGTCTGACCCACTGTA & GGATCAGCTCCAGCCACATT \\
\hline SREBF1 & ACAGTGACTTCCCTGGCCTAT & GCATGGACGGGTACATCTTCAA \\
\hline BMl1 & AGTTCCACTCTGCCTTCAGC & CATTGTCTCGCCCCGATCTC \\
\hline RING1A & CATTGTCTCGCCCCGATCTC & GATTGCTGTTTCCCCTCGGT \\
\hline RING1B & CGGGGAGAGGCGATGCTATT & TACCCAAAGCCTTCACACCAC \\
\hline ELOVL2 Promoter & ATCAGTTCGGATAACGGCCC & TAGAAGCGCAGGCTCTAGGA \\
\hline \multicolumn{3}{|l|}{ siRNA sequence } \\
\hline BMI1-siRNA-1 & CCUAAUACUUUCCAGAUUGAU tt & AUCAAUCUGGAAAGUAUUACGG tt \\
\hline BMI1-siRNA-2 & CGGAAAGUAAACAAAGACAAA tt & UUUGUCUUUGUUUACUUUCCG tt \\
\hline RING1A-siRNA-1 & GCCCUGAUCUCUAAGAUCUAU tt & AUAGAUCUUAGAGAUCAGGGC tt \\
\hline RING1A-siRNA-2 & AGACGAGGUAUGUGAAGACAA $\mathrm{t} t$ & UUGUCUUCACAUACCUCGUCU tt \\
\hline RING1B-siRNA-1 & GCCAGCAUCAACAAGCACAAU tt & AUUGUGCUUGUUGAUCCUGGC tt \\
\hline RING1B-siRNA-2 & GGAGUGUUUACAUCGUUUU tt & AAAACGAUGUAAACACUCC $t \mathrm{t}$ \\
\hline \multicolumn{3}{|l|}{ shRNA sequence } \\
\hline MYCN-shRNA-1 & \multicolumn{2}{|c|}{ CCGGGCCAGTATTAGACTGGAAGTTCTCGAGAACTTCCAGTCTAATACTGGCTIIITGAATTC } \\
\hline MYCN-shRNA-2 & \multicolumn{2}{|c|}{ CCGGCTGAGCGATTCAGATGATGAACTCGAGTTCATCATCTGAATCGCTCAGTTITTGAATTC } \\
\hline ELOVL2-shRNA-1 & \multicolumn{2}{|c|}{ CCGGGGTGCTTTGGTGGTACTATTTCTCGAGAAATAGTACCACCAAAGCACCTITTGAATT } \\
\hline ELOVL2-shRNA-2 & \multicolumn{2}{|c|}{ CCGGTATGTTTGGACCGCGAGATTCCTCGAGGAATCTCGCGGTCCAAACATATTITTAATT } \\
\hline
\end{tabular}

to the above solution. The bi-phasic solution was vigorously mixed for $30 \mathrm{~s}$ and separated into two phases by centrifugation at $3000 \mathrm{rpm}$ for $5 \mathrm{~min}$. The upper isooctane phase containing the free fatty acid (FFA) fraction was collected and evaporated to dryness. For GC-MS, an Agilent $6890 \mathrm{~N}$ gas chromatograph equipped with an Agilent 7683 autosampler (Santa Clara) was utilized and operated along with an Agilent 5975 mass selective detector in negative ion mode using NCI with methane as the reagent gas.

\section{Elisa}

A DHA ELISA Kit (purchased from Cloud-Clone Corp) was used to measure the DHA concentration. Then, $50 \mu \mathrm{L}$ of dilutions of the standard, blank and cell lysates were added into the appropriate wells, and $50 \mu \mathrm{L}$ of Detection Reagent A was added to each well immediately. The plate was incubated for $1 \mathrm{~h}$ at $37^{\circ} \mathrm{C}$. The solution was aspirated and washed with $350 \mu \mathrm{L}$ of $1 \mathrm{X}$ Wash Solution to each well 3 times. Then, $100 \mu \mathrm{L}$ of Detection
Reagent B working solution was added and incubated for $30 \mathrm{~min}$ at $37^{\circ} \mathrm{C}$. The solution was aspirated and washed with $350 \mu \mathrm{L}$ of $1 \mathrm{X}$ Wash Solution to each well 3 times. Next, $90 \mu \mathrm{L}$ of Substrate Solution was added to each well and incubated for $15 \mathrm{~min}$ at $37^{\circ} \mathrm{C}$, followed by the addition of $50 \mu \mathrm{L}$ of Stop Solution to each well. The liquid turned yellow after the addition of Stop solution. The microplate reader was immediately used to conduct measurements at $450 \mathrm{~nm}$.

\section{Cell growth assay}

A CCK-8 colorimetric assay was used to measure the cell growth and viability, as described previously [42]. Briefly, $2 \times 10^{3}$ cells per well were seeded into a flatbottomed 96-well microtiter plate and incubated with either solvent control (0.1\% DMSO) or various concentrations of DHA. At $0,24,48$, and $72 \mathrm{~h}$, spent medium was carefully removed and $100 \mu \mathrm{L}$ DMEM containing $10 \%$ CCK-8 solution (TaKaRa) was added to each well and incubated for $3 \mathrm{~h}$ at $37^{\circ} \mathrm{C}$ with $5 \% \mathrm{CO} 2$, the relative 
cell number was recorded by a Benchmark Microplate Reader at $450 \mathrm{~nm}$ (Bio-Rad Laboratories).

\section{Cell cycle analysis}

The cell cycle profile of the treated BE(2)-C, IMR-32 and SK-N-AS cells was analysed by flow cytometry. A total of $1 \times 10^{5}$ treated cells per well were seeded into a 6-well plate dish and incubated overnight. On the day of analysis, the cells were permeabilized with $70 \%$ ethanol at $4{ }^{\circ} \mathrm{C}$ for $30 \mathrm{~min}$. Subsequently, the cells were treated with propidium iodide (PI) and analysed by flow cytometry (FACSCanto flow cytometer; BD Biosciences) for cell cycle distribution using the ModFit LT V3.0 software (Verity Software House, Topsham).

\section{Western blot analysis}

Cells were lysed for Western blotting in RIPA buffer (Cell Signaling Technology) with complete protease inhibitor cocktail (Roche). For the detection of histone H3 acetylation levels, cells were lysed in SDS lysis buffer (62.5 mM Tris- $\mathrm{HCl}, 2 \%$ SDS, 10\% (v/v) glycerol, $1 \mathrm{mM}$ DTT and complete protease inhibitor cocktail). The following antibodies were used: anti-MYCN (B8.4.B, sc-53, 993, Santa Cruz), anti-ELOVL2 (EPR11880, ab176327, Abcam), anti-monoubiquitin H2A (Lys119) (ABE569, Millipore), anti-BMI1 (6964, Cell Signaling Technology), anti-RING1A (2820, Cell Signaling Technology), antiRING1B (5694, Cell Signaling Technology), anti-SREBP1 (sc-13,551, Santa Cruz Biotechnology), and anti-ß3-actin (clone AC-15, Sigma-Aldrich).

\section{RNA extraction and qRT-PCR}

The RNeasy Mini Kit (Qiagen) was used to extract total RNA from cell lines or snap-frozen xenograft tissue. The TaKaRa PrimeScript RT reagent kit was used to transcribe cDNA for qRT-PCR analysis. The relative gene expression was measured using SYBR Green qPCR Master Mix (Perkin-Elmer Applied Biosystems) on an ABI Flex 6 thermal cycler (Perkin-Elmer Applied Biosystems) and normalized to the averaged expression of $18 \mathrm{~S}$ mRNA. The primers used in qRT-PCR are listed in Table 1. Data were analysed using Applied Biosystems QuantStudio $^{\text {Tm }}$ Real-Time PCR Software, and changes in expression were calculated using the $\Delta \Delta \mathrm{CT}$ method for cell lines.

\section{ChIP}

A total of $1 \times 10^{6}$ cells were collected and lysed for ChIP in buffer containing $50 \mathrm{mM}$ Tris- $\mathrm{HCl}, \mathrm{pH} 8.1,1 \%$ SDS, $10 \mathrm{mM}$ EDTA and complete protease inhibitor cocktail (Roche), then sonicated to obtain 200-1000 bp DNA fragments. ChIP was performed according to the ChIP Assay Kit (Millipore) protocol. The following antibodies were used: anti-MYCN (B8.4.B, sc-53,993, Santa Cruz), anti-acetyl-histone H3 (Lys9) (7-352, Millipore), antitrimethyl-histone H3 (Lys4) (07-473, Millipore), antimonoubiquitin H2A (Lys119) (ABE569, Millipore), antiBMI1 (6964, Cell Signaling Technology), anti-RING1A (2820, Cell Signaling Technology), anti-RING1B (5694, Cell Signaling Technology), anti-SREBP1 (sc-13,551, Santa Cruz Biotechnology), normal mouse IgG (sc-2025, Santa Cruz) and normal rabbit IgG (sc-2027, Santa Cruz). Primers for specific qRT-PCR amplification of the ELOVL2 promoter region were: forward: 5 '-ATCAGT TCGGATAACGGCCC-3', reverse: 5' - TAGAAGCGCA GGCTCTAGGA-3'.

ChIP sequencing data of ELOVL2 promoter interactions with MYCN and H3K4me3 in NGP, SK-N-BE and SK-N-SH NB cell lines were achieved from Gene Expression Omnibus (GSM2113529, GSM2113519 and GSM2308437), and the analysis was performed online in the Cistrome platform (http://cistrome.org).

\section{Co-immunoprecipitation (co-IP) assays}

Co-IP assays were performed to identify the proteins. Briefly, $1 \times 10^{6}$ cells were collected and lysed for IP-MS in $300 \mu \mathrm{L}$ buffer containing $1 \% \mathrm{NP} 40,150 \mathrm{nM} \mathrm{NaCl}, 50$ $\mathrm{mM}$ Tris-Cl (pH 7.2), $1 \mathrm{mM}$ EDTA nuclear extracts (NE) and complete protease inhibitor cocktail (Roche). For Co-IP using antibodies, before being added to the cell lysates, the antibodies were incubated with Protein $\mathrm{A} / \mathrm{G}$ Magnetic Beads (Millipore) for $3 \mathrm{~h}$ at $4{ }^{\circ} \mathrm{C}$. Then, the crosslinked Protein A/G Magnetic Beads (Millipore) were added to the cell lysates directly and incubated overnight at $4{ }^{\circ} \mathrm{C}$. The magnetic beads were washed with IP wash buffer and collected. The protein complexes were eluted from the beads by $50 \mathrm{mM}$ glycine ( $\mathrm{pH} 2.8$ ) and analysed by MS.

\section{Animal experiments}

$\mathrm{BE}(2)-\mathrm{C}$ cells were transfected for $96 \mathrm{~h}$ with empty, MYCN and/or ELOVL2 vectors in culture, and SK-NAS cells were transfected for $72 \mathrm{~h}$ with the negative control or shRNA targeting MYCN and/or ELOVL2 vectors in culture; then, $2 \times 10^{6}$ viable cells suspended in $200 \mu \mathrm{l}$ Basement Matrigel (BD Biosciences) were subcutaneously implanted in the flanks of 4-week-old female nude mice ( $n=5$ per study group). Tumor size was measured with a calliper every 3 or 4 days. After 24 days, the animals were sacrificed, and the tumor volume was calculated by $\pi / 6(\mathrm{~s} 1 \times \mathrm{s} 2 \times \mathrm{s} 2)$, where $\mathrm{s} 1$ was the largest tumor diameter, and s2 was the smallest tumor diameter. Experiments conformed to the regulatory standards and were approved by the local ethics committee.

\section{Statistical analysis}

Kaplan-Meier analyses and comparison of ELOVL2 expression between different patient subgroups for the 496 
patients neuroblastoma cohort were performed online in the R2 platform (http://r2.amc.nl), and the resulting survival curves, box plots and $P$ values (log-rank test) were downloaded. The results of the cell culture experiments were compared using the one-sample t-test in GraphPad Prism version 5.0 (GraphPad Software Inc., La Jolla, CA) unless otherwise indicated. $\mathrm{P}$ values below 0.05 were considered significant.

\section{Results}

\section{MYCN negatively regulates DHA synthesis via ELOVL2}

To identify the potential role of MYCN in FA metabolism regulation, we first used GC-MS to profile the medium- and long-chain FA landscape after MYCN depletion in the MYCN-amplified neuroblastoma cells IMR32. IMR32 cells were infected with the lentivirus expressing 2 shRNAs targeting MYCN or the negative control for $72 \mathrm{~h}$ before GC-MS profiling. MYCN depletion resulted in significant upregulation of various types of FAs (Fig. 1a), of which DHA was the most strongly upregulated with a 1.6- to 1.61-fold induction. ELISA analysis validated that DHA was dramatically upregulated (3.1- to 3.2- fold in IMR32 and 2.9- to 3.6- fold in another MYCN-amplified neuroblastoma cell line, BE(2)-C cells (Fig. 1b). Considering that the strongest DHA induction by MYCN depletion occurred in MYCNamplified cells, we speculated that the endogenous DHA content are different in neuroblastoma cell lines with different MYCN genomic statuses. As shown in Fig. 1c, the MYCN-amplified cell lines $\mathrm{BE}(2)-\mathrm{C}$ and IMR32 expressed distinctly lower DHA levels than SK-N-AS cells, which maintained a single MYCN copy. Furthermore, enforced MYCN expression reduced endogenous DHA levels in MYCN-nonamplified SK-N-AS cells (Fig. 1d). We next tested the direct influence of DHA on cell growth by a CCK- 8 assay. After DHA treatment, IMR32 and $\mathrm{BE}(2)-\mathrm{C}$ cells exhibited lower proliferation rates in a DHA concentration-dependent manner (Additional file 1: Figure S1A). Meanwhile, the cell cycle profiles of IMR32 and $\mathrm{BE}(2)-\mathrm{C}$ cells were examined by flow cytometry. DHA treatment caused cell cycle arrest at the G0/G1 phase (Additional file 1: Figure S1B). Moreover, DHA treatment reduced the colony formation of two cells in soft agar (Additional file 1: Figure S1C). Given that, MYCN suppresses DHA transporter or synthesis to favor tumor cell growth.

To identify the metabolic activity causally involved, eight known DHA synthesis and transporter genes were detected by $\mathrm{qPCR}$ in $\mathrm{BE}(2)-\mathrm{C}$ and IMR32 cells, which expressed low levels of MYCN after shRNA-mediated knockdown using two different shRNAs. MYCN depletion most strongly induced ELOVL2 expression in both cells, resulting in a 3.9- to 7.3-fold induction in IMR32 cells and a 3.9- to 5.5-fold induction in BE(2)-C cells
(Fig. 1e and f). ELOVL7 increased 2.2 (ns)- to 6.0-fold in IMR32 cells and 1.4- to 1.5-fold in $\mathrm{BE}(2)$-C cells (Fig. 1 e and f). Meanwhile, depletion of MYCN slightly downregulated SLC27A2 and FADS2 in IMR32 cells and did not affect the expression of ELOVL4, ELOVL5, SLC27A3 or FADS1 (Fig. $1 \mathbf{e}$ and $\mathbf{f}$ ). In line with the mRNA expression level after MYCN depletion, the protein level of ELOVL2 was also upregulated in both neuroblastoma cell lines (Fig. 1j). Importantly, by using the SEQC-498 cohort, we performed a differential RNA expression analysis of the MYCN-amplification and nonamplification neuroblastoma tumors. Of all seven known DHA synthesis and transporter genes, the decrease of ELOVL2 expression was mostly significant in MYCN amplified neuroblastoma (Fig. 1g). We further reanalysed RNA-sequencing data from a series of neuroblastomas cohort and draw the same conclusion that ELOVL2 expression was significantly correlated with MYCN status (Additional file 2: Figure S2). In line with clinical tumor samples, the MYCN-amplified cell lines $\mathrm{BE}(2)-\mathrm{C}$ and IMR32 expressed lower ELOVL2 expression than MYCN-nonamplified cell lines SK-N-AS cells (Fig. 1h), and the protein level of ELOVL2 was vilified in Fig. 1j. Enforced MYCN expression reduced ELOVL2 expression in MYCN-nonamplified SK-N-AS cells (Fig. 1i), and the protein level of ELOVL2 was vilified in Fig. 1j. In conclusion, our data showed that MYCN represses DHA synthesis via ELOVL2 inhibition.

ELVOL2 is critical for DHA accumulation and proliferation inhibition in neuroblastoma cells

To test the biological effects of ELOVL2 in MYCNamplified neuroblastoma cells, ELOVL2 was sustainably overexpressed in MYCN-amplified BE(2)-C and IMR32 cells by a lentivirus expressing ELOVL2. Transfection efficacy was validated by assessing the expression of ELOVL2 by qPCR and western blot (Additional file 3: Figure S3). Enforced ELOVL2 expression increased the DHA content up to 1.4- and 1.5-fold in IMR32 and $\mathrm{BE}(2)-\mathrm{C}$ cells, respectively (Fig. 2a), and reduced the proliferation rate of both cells (Fig. $2 \mathrm{~b}$ and c). The cell cycle profiles of IMR32 and BE(2)-C were examined by flow cytometry. Enforced ELOVL2 expression caused cell cycle arrest at the G0/G1 phase, accompanied by a decrease in the percentage of cells in the $\mathrm{S}$ phase, especially in IMR32 cells (Fig. 2d and e). Moreover, enforced ELOVL2 expression reduced the colony formation of these two cell lines in soft agar (Fig. 2f, g and h).

SK-N-AS Cells with a single copy of MYCN endogenously express higher ELOVL2 levels. Hence, we knocked down ELOVL2 in SK-N-AS cells with two different shRNAs. Transfection efficacy was validated by assessing the expression of ELOVL2 by qPCR (Additional file 3: Figure S3). ELOVL2 knockdown decreased the DHA content (Fig. 2i), increased the proliferation rate (Fig. 2j), 


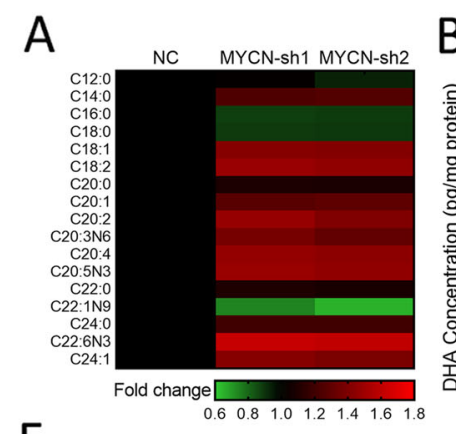

$\mathrm{E}$

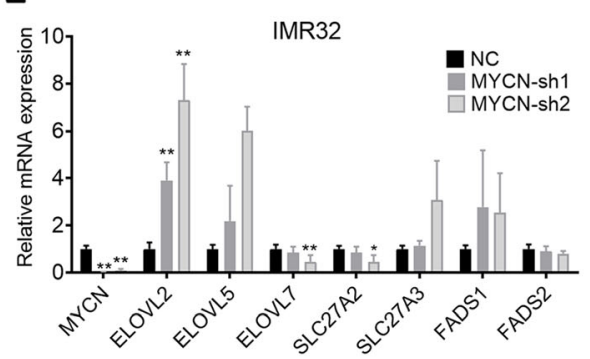

G

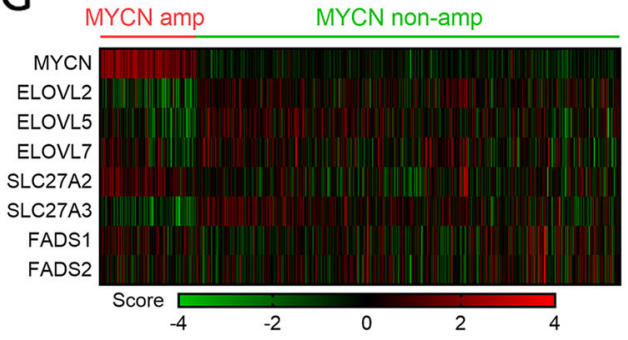

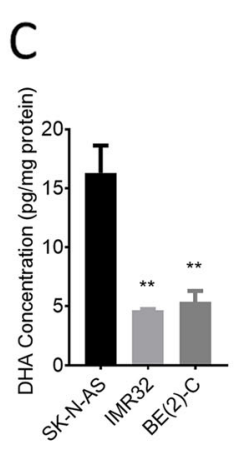

$\mathrm{F}$

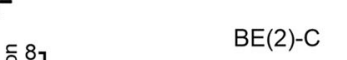

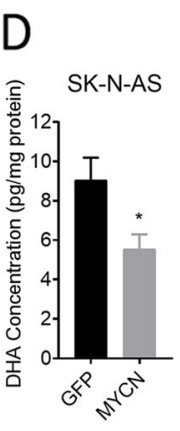

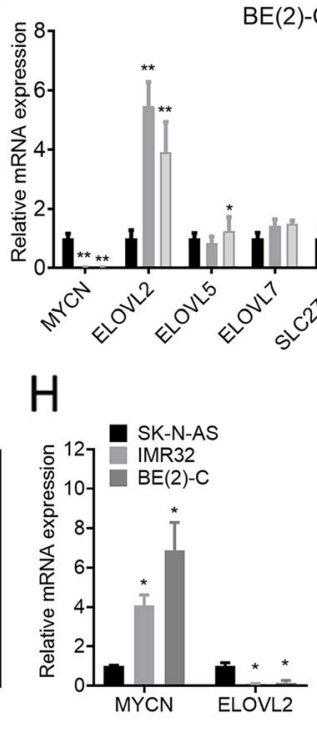

NC

MYCN-sh1

$\square \mathrm{MYCN}-\mathrm{sh} 2$

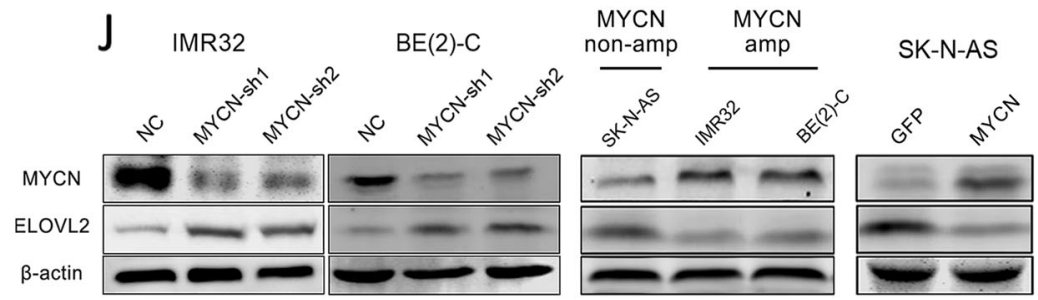

Fig. 1 MYCN RNAi strongly upregulated DHA and ELOVL2 in neuroblastoma cell lines. a Heatmap representation of FA profiling indicates significantly $(P<0.05)$ up- (red) or downregulated (green) FAs in BE(2)-C cells treated for $72 \mathrm{~h}$ with the lentivirus expressing 2 MYCN shRNA or the negative control. $\mathbf{b}$ IMR32 and BE(2)-C cells were treated as described in (a). The change in DHA concentration was validated using ELISA. c DHA concentration of MYCN-amplified cell lines (IMR32 and BE(2)-C) and MYCN single-copy cell lines SK-N-AS were measured by ELISA. d The DHA concentration of SK-N-AS cells stably expressing the GFP control or MYCN was measured by ELISA. e and $\mathbf{f} I M R 32$ and BE(2)-C stably expressing negative control or MYCN shRNA. The change in DHA transport and synesis-related mRNA expression was measured by qRT-PCR (fold-change over negative control \pm SD). $\mathbf{g}$ RNA expression pattern of ELOVL2 with MYCN amplified verse MYCN nonamplified in a cohort of 476 neuroblastoma. $\mathbf{h}$ MYCN and ELOVL2 mRNA expression of MYCN-amplified cell lines (IMR32 and BE(2)-C) and MYCN single-copy cell lines (SK-NAS) were measured by qRT-PCR (fold-change over SK-N-AS \pm SD). i MYCN and ELOVL2 mRNA expression of SK-N-AS cells stably expressing GFP control or MYCN were measured by qRT-PCR (fold-change over GFP control \pm SD). $\mathbf{j}$ Using an MYCN and ELOVL2 antibody with the ß-actin loading control. MYCN RNAi efficiency and the change in ELOVL2 expression was validated by Western blots (left). The difference of MYCN and ELOVL2 between MYCN-amplified cell lines (IMR32 and BE(2)-C) and MYCN single-copy cell lines (SK-N-AS) was validated by Western blots (middle). MYCN overexpression efficiency and the change in ELOVL2 expression were validated by Western blots (right). ${ }^{*} P<0.05 ;{ }^{*} P<0.01$

decreased cell cycle arrest at the G0/G1 phase (Fig. 2k), and increased colony formation in soft agar (Fig. $2 \mathrm{l}$ and $\mathrm{m}$ ); re-supplementation with $50 \mathrm{nM}$ DHA partly counteracted the pro-proliferation effect induced by ELOVL2 depletion.
Moreover, re-supplementation with other kinds of PUFA, including $50 \mathrm{nM}$ LA (linoleic acid), ALA ( $\alpha$-Linolenic acid), AA (Arachidonic acid) or DPA(n-6) (Docosapentaenoic Acid n-6) did not counteracted the pro-proliferation effect 

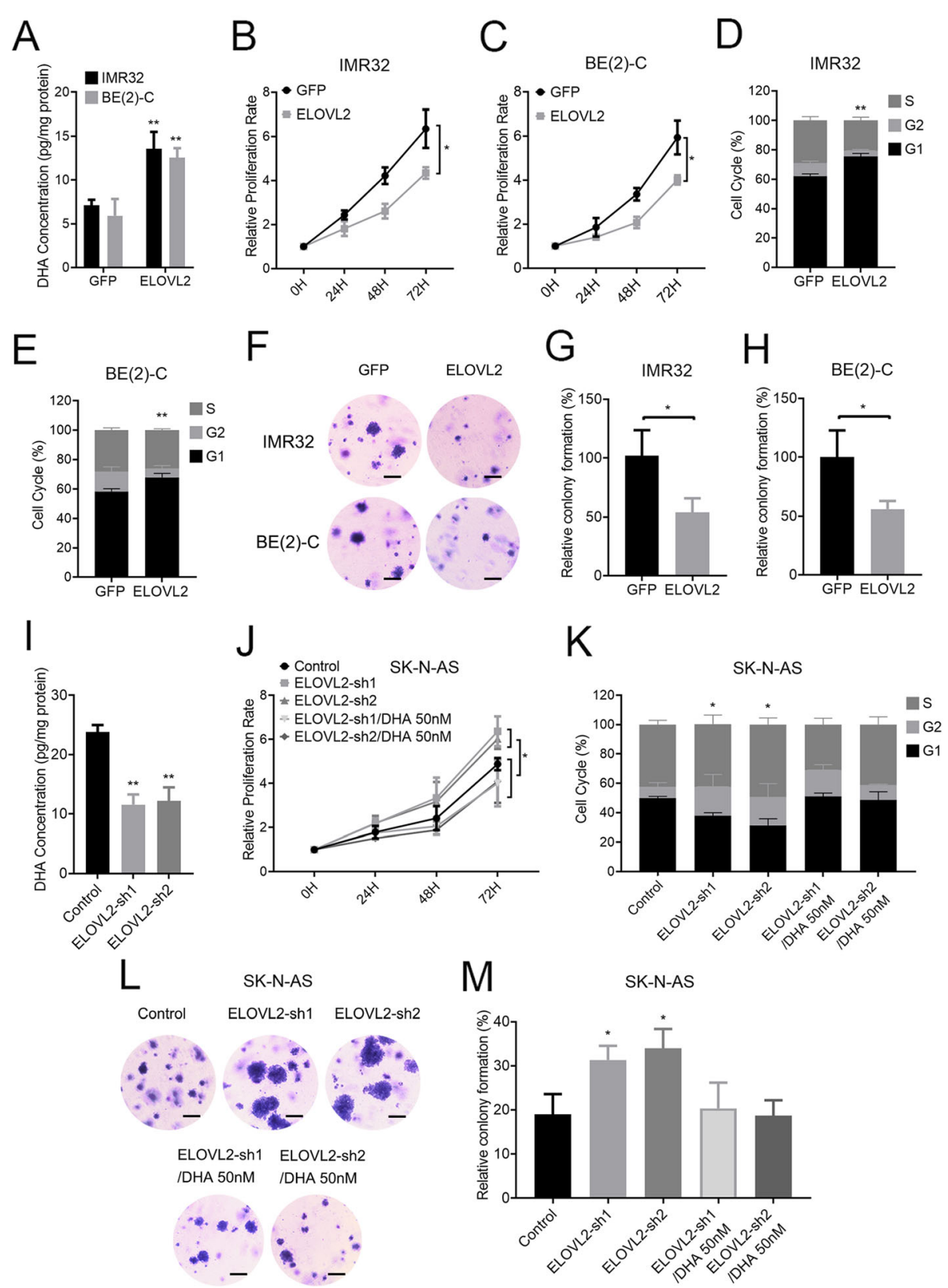

Fig. 2 ELOVL2 inhibits cell proliferation in vitro. a Relative DHA concentrations of IMR32 and BE(2)-C cells stably expressing GFP or ELOVL2 were measured by ELISA (fold-change over GFP control \pm SD). $\mathbf{b}$ and $\mathbf{c}$ The proliferation rate of IMR32 and BE(2)-C cells stably expressing GFP or ELOVL2 was assayed using CCK-8 and a spectrophotometer at OD450. $\mathbf{d}$ and e IMR32 and BE(2)-C cells stably expressing GFP or ELOVL2 were stained with PI for $72 \mathrm{~h}$, and the change in the cell cycle was measured by flow cytometry. $\mathbf{f}$ IMR32 and BE(2)-C stably expressed GFP or ELOVL2. Colony growth in soft agar is shown for representative cultures after staining with crystal violet. Scale bar, $25 \mu \mathrm{m}$. $\mathbf{g}$ and $\mathbf{h}$ The results of the soft agar assays of $(\mathbf{f})$ are presented as bar graphs of the mean number of colonies ( \pm SD) forming. i Relative DHA concentration of SK-N-AS cells stably expressing the negative control or ELOVL2 shRNA was measured by ELISA (fold-change over negative control \pm SD). $\mathbf{j}$ SK-N-AS cells stably expressing the negative control or ELOVL2 shRNA were treated with DHA $50 \mathrm{nM}$. The proliferation rate was assayed using CCK-8 and a spectrophotometer at OD450. $\mathbf{k}$ SK-N-AS cells stably expressing the negative control or ELOVL2 shRNA were stained with PI for $72 \mathrm{~h}$, and the change in the cell cycle was measured by flow cytometry. I SK-N-AS cells stably expressed the negative control or ELOVL2 shRNA. Colony growth in soft agar is shown for representative cultures after staining with crystal violet. Scale bar, $25 \mu \mathrm{m}$. $\mathbf{m}$ The results of the soft agar assays of $(\mathrm{L})$ are presented as bar graphs of the mean number of colonies ( \pm SD) forming. ${ }^{*} P<0.05$; ${ }^{* *} P<0.01$ 
induced by ELOVL2 depletion, and re-supplementation with $50 \mathrm{nM}$ EPA (Eicosapentaenoic Acid) partly counteracted the pro-proliferation effect induced by ELOVL2 depletion (Additional file 4: Figure S4). Therefore, ELOVL2 possesses anti-tumor properties in neuroblastoma cells via DHA synthesis.

\section{MYCN transcriptionally regulates ELOVL2 expression via PRC1-mediated H2AK119ub in neuroblastoma cells}

Protein binding profiles from the Gene Expression Omnibus ChIP-seq database revealed that MYCN could bind to the ELOVL2 promoter in the MYCN-amplified cell line NGP, and histone 3 lysine 4 tri-methylation (H3K4me3) was enriched at the ELOVL2 promoter region in the MYCN single-copy cell line SK-N-SH but not in the MYCN-amplified cell line BE(2)-C (Fig. 3a). Further ChIPqPCR analysis confirmed the Cistrome database results, which showed abundant enrichment of MYCN at the ELOVL2 promoter in BE(2)-C and IMR32 cells (Fig. 3b), increased H3K4me3 and H3K9ac, and reduced H3K27me3 upon MYCN knockdown in BE(2)-C cells (Fig. 3c). Next, we aimed to decipher events occurring at the ELOVL2 promoter region. An immunoprecipitation-mass spectrometry (IP-MS) approach was used to identify interactive proteins with MYCN, which identified a small set of transcription factors, including RING1A, RING1B and BMI1, three major components of polycomb repressive complex 1 (PRC1) (Fig. 3d). To verify the results of IP-MS, we tested whether the MYCN, RING1A, RING1B and BMI1 proteins physically cooperate by Western blots in BE(2)-C cells. IP with an antibody against MYCN showed that the enrichment of RING1A, RING1B and BMI1 were significantly higher in the anti-MYCN group than in the IgG control group (Fig. 3e). IP with an antibody against RING1A, RING1B and BMI1 showed an increased enrichment of MYCN above that in the IgG control (Fig. 3f). To verify PRC1 components involved in the transcriptional repression of ELOVL2, we transiently knocked down RING1A, RING1B and BMI1 using two different siRNAs to eliminate nonspecific and off target effects in $\mathrm{BE}(2)-\mathrm{C}$ cells, and we then assessed ELOVL2 expression. Transfection efficacy was validated by qPCR (Additional file 4: Figure S4C). Depletion of RING1A did not affect ELOVL2 expression. RING1B and BMI1 depletion induced ELOVL2 expression 2.0- to 2.1-fold and 3.3- to 3.7-fold $72 \mathrm{~h}$ after knockdown, respectively (Fig. 3g). To test whether the PRC1 complex was recruited to this ELOVL2 promoter region and led to H2AK119ub (histone 2A lysine 119 monoubiquitination), we performed ChIP-qPCR. RING1B and BMI1 were recruited to this region, and H2AK119ub enrichment was elevated (Fig. 3h). To analyse whether MYCN is required for PRC1 complex-mediated H2AK119ub at the ELOVL2 promoter, we performed ChIP-qPCR. Stable MYCN knockdown in $\mathrm{BE}(2)-\mathrm{C}$ cells decreased the recruitment of
RING1B and BMI1 to the ELOVL2 promoter by 90\% and $\sim 60 \%$, respectively, reducing H2AK119ub enrichment by $\sim 80 \%$ (Fig. 3h). RING1B or BMI1 knockdown did not decrease the enrichment of MYCN to the ELOVL2 promoter (Fig. 3i). SREBP1 was a known transcription factor that activated ELOVL2 expression [43], which was further verified by ChIP-qPCR, a luciferase assay and a Western blot in the neuroblastoma cells SK-N-AS (Additional file 5: Figure S5). MYCN, RING1B and BMI1 knockdown increased the recruitment of SREBP1 to the ELOVL2 promoter by 7-, 3.5- and 2.5-fold, respectively (Fig. 3j). In conclusion, MYCN recruits the PRC1 complex, cooccupies the ELOVL2 promoter, mediates H2AK119ub and suppresses its transcription in neuroblastoma cells.

\section{ELVOL2 inhibits tumor progression in vivo and predicts favorable survival}

Analysis of mouse xenografts using SK-N-AS cells that stably expressed shRNA targeting ELOVL2 showed that knockdown of ELOVL2 significantly enhanced tumor volume (Fig. 4a and b), mass (Additional file 6: Figure S6A) and decreased the DHA content (Fig. 4c) compared to those in the nontargeting control group. On the other hand, analysis of mouse xenografts using $\mathrm{BE}(2)-\mathrm{C}$ cells that stably expressed ELOVL2 showed that compared to the GFP control group, the enforced ELOVL2 expression group had a significantly reduced tumor volume (Fig. $4 \mathrm{~d}$ and e), mass (Additional file 6: Figure S6B) and an increased DHA content (Fig. 4f).

To further explore the clinical feature of ELOVL2 in neuroblastoma, we assessed whether ELOVL2 is differentially expressed in primary tumors. By IHC analysis of a tissue chip, we compared the difference in ELOVL2 protein expression in normal nerve tissues and neuroblastoma specimens with different international neuroblastoma staging system (INSS) stages. ELOVL2 expression is relatively lower in neuroblastoma than in normal nerve tissues and negatively correlated with INSS stage (Fig. $4 \mathrm{~g}$ and $\mathrm{h}$ ). The detailed patient information was provided in Additional file 7: Table S1. We reanalysed RNA-seq data from a cohort of 496 neuroblastomas [44]. Kaplan-Meier analysis showed that high-level ELOVL2 expression in tumors correlated both with favorable overall and event-free patient survival (Fig. $4 \mathrm{i}$ and $\mathrm{j}$ ). Furthermore, at the transcriptional level, high-level ELOVL2 expression also significantly correlated with established clinical and molecular markers for favorable tumor biology, including INSS 1, 2 or 4S stage disease (Fig. 4k), Single-copy MYCN status (Fig. 4l), age at diagnosis $\leq 18$ months (Fig. $4 \mathrm{~m}$ ), a low-risk tumor transcriptional profile defined by principal access method (PAM) analysis (Fig. 4n) and favorable Shimada/INPC tumor histology (Fig. 4o). Taken together, ELOVL2 inhibits neuroblastoma growth in vivo and could be regarded as a favorable marker. 

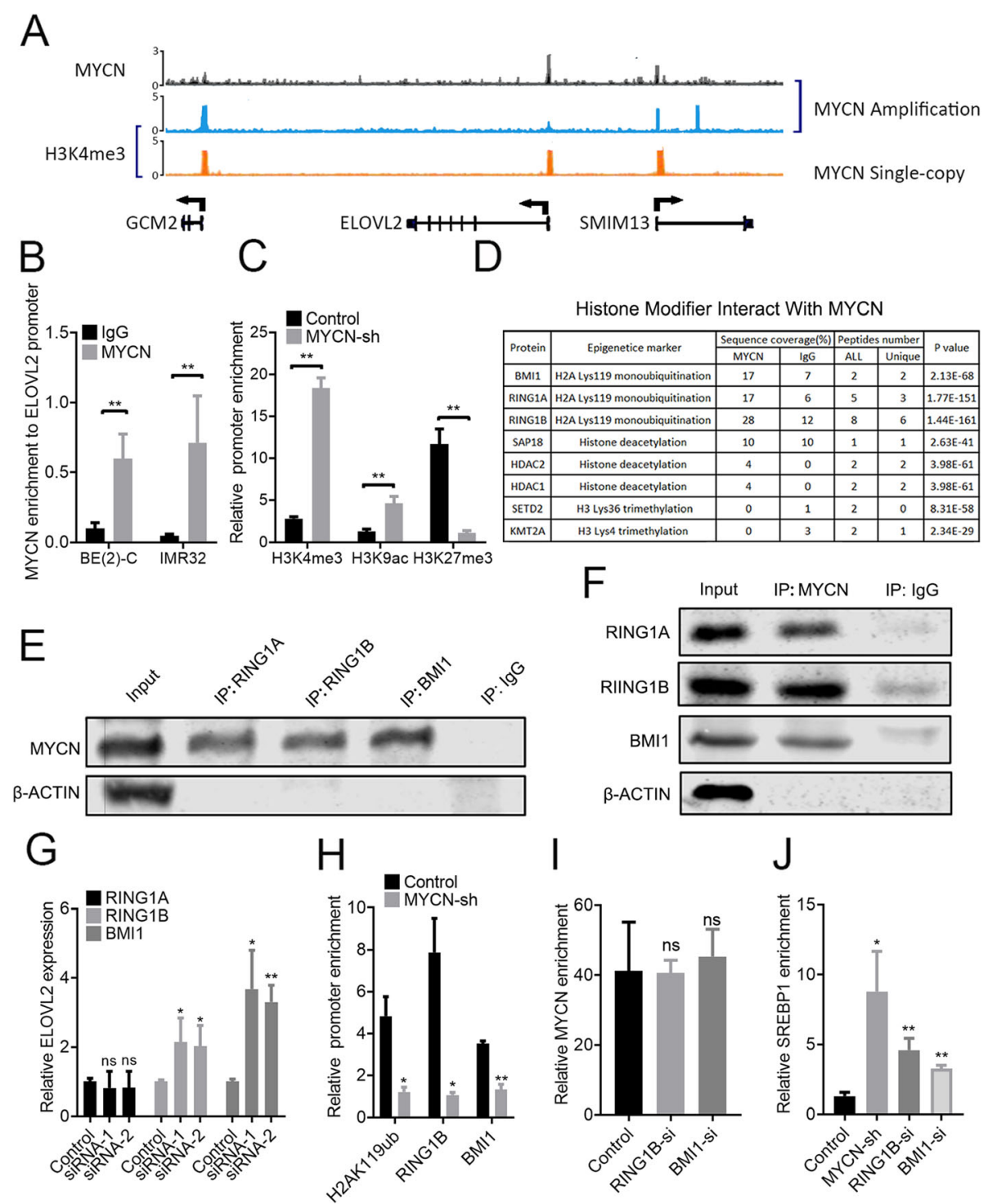

Fig. 3 MYCN recruits the PRC1 complex to the ELOVL2 promoter region and mediates ELOVL2 inhibition by H2AK1 19ub. a ChIP-Seq analysis from MYCN-amplified cell lines (NGP and BE(2)-C) and MYCN single-copy cell lines (SK-N-SH) using antibodies detecting MYCN (black bar) and H3K4me3 (blue and orange bar) superimposed on the ELOVL2 cluster genomic organization. b ChIP analysis in the IMR32 and BE(2)-C cell lysates showing MYCN recruitment to the ELOVL2 promoter region. c ChIP analysis in BE(2)-C cells stably expressing the negative control or ELOVL2 shRNA showing reduced H3K4me3 and H3K9ac enrichment, and increased H3K27me3 enrichment to the ELOVL2 promoter region. d BE(2)-C cell lysates immunoprecipitated with antibodies against MYCN were analysed using mass spectrometry to detect potential binding partner proteins of MYCN. The PRC1 complex consisting of BMI1, RING1A and RING1B was detected. $\mathbf{e}$ and $\mathbf{f}$ Western blots of BE(2)-C cell lysates coimmunoprecipitated with antibodies against MYCN, BMI1, RING1A and RING1B shown with the IgG control antibody and 1\% of the lysate input to assess the PRC1-MYCN protein interaction. $\mathbf{g}$ qRT-PCR showing relative ELOVL2 mRNA expression (fold-change over negative control \pm SD) in BE(2)-C cells $48 \mathrm{~h}$ after BMI1, RING1A or RING1B siRNA treatment. $\mathbf{h}$ ChIP analysis showing relative ELOVL2 promoter enrichment of H2AK119ub, RING1B and BMI1 in BE(2)-C cells treated as in (B). i ChIP analysis showing relative ELOVL2 promoter enrichment of MYCN in BE(2)-C cells $48 \mathrm{~h}$ after BMI1 or RING1B siRNA treatment. $\mathbf{j}$ ChIP analysis showing relative ELOVL2 promoter enrichment of SREBP1 in BE(2)-C cells treated as in (b) and (i). ${ }^{*} P<0.05$; ${ }^{* *} P<0.01$

MYCN regulates tumor progression via ELOVL2 repression in vitro and in vivo

To further confirm the repression of ELOVL2 in MYCN-induced cell proliferation, we knocked down ELOVL2 in MYCN-depleted BE(2)-C cells, which resulted in re-increased cell proliferation (Fig. 5a) and cell cycle acceleration (Fig. 5c). Mouse xenograft results further showed that ELOVL2 knockdown again reenhanced tumor growth (Fig. 5e and f), mass (Additional file 6: Figure S6C) and re-decreased the DHA content in tumors (Fig. 5g). In contrast, enforced ELOVL2 expression in MYCN-overexpressing SK-N-AS cells showed 


\section{A}

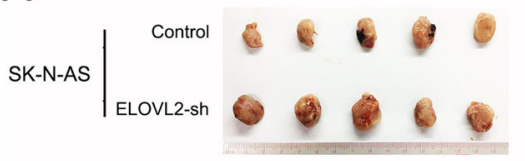

B

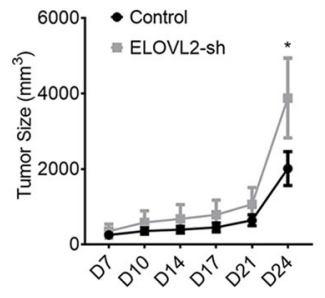

$\mathrm{C}$
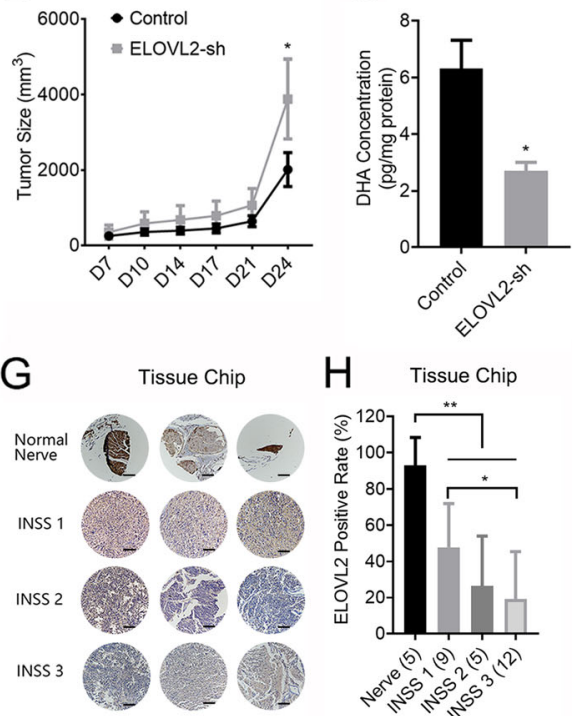

$\mathrm{D}$

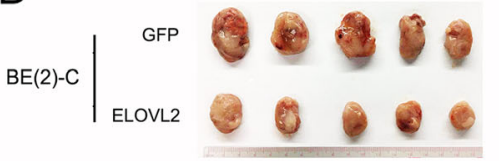

$\mathrm{E}$

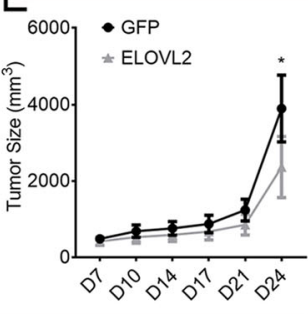

$\mathrm{F}$

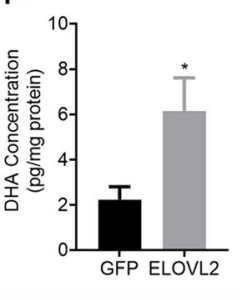

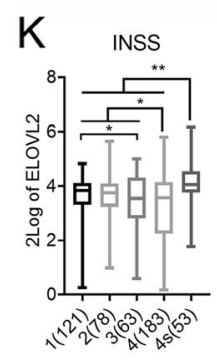
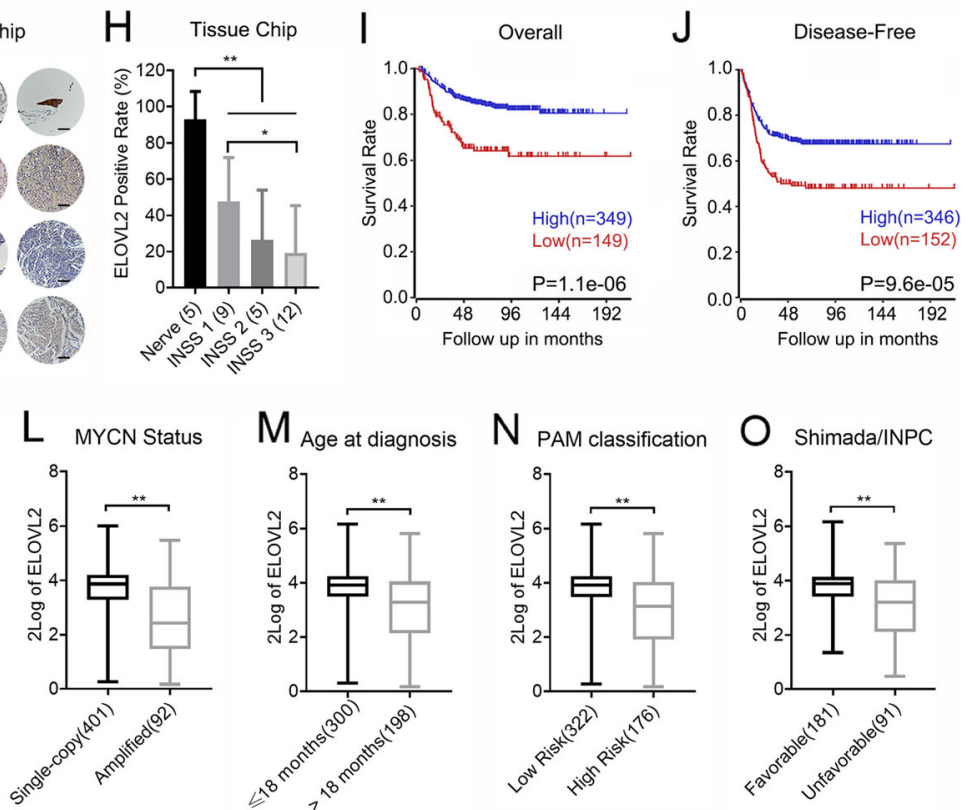

Fig. 4 ELOVL2 suppresses tumor growth in vivo and correlates with favorable neuroblastoma patient survival. a, b and c SK-N-AS cells stably expressing negative control or ELOVL2 shRNA were injected subcutaneously into nude mice ( $n=5$ for each group). Tumors were compared (a) at the end of the experiment. Tumor growth curves (b) were measured starting at 7 days after inoculation. The DHA concentration of tumors (c) was measured by ELISA. $\mathbf{d}$, e and $\mathbf{f} B E(2)-C$ cells stably expressing ELOVL2 or the GFP control were injected subcutaneously into nude mice ( $n=5$ for each group). Tumors were compared (d) at the end of the experiment. Tumor growth curves (e) were measured starting at 7 days after inoculation. The DHA concentration of tumors (f) was measured by ELISA. g Representative IHC analysis of ELOVL2 protein expression in normal nerve tissues and neuroblastoma specimens of different INSS stages (1-3) is shown. Scale bar, $100 \mu \mathrm{m}$. $\mathbf{h}$ The results of IHC analysis of ELOVL2 expression are presented as bar graphs of the mean number of the ELOVL2-positive rate. $\mathbf{i}$ and $\mathbf{j}$ Overall (i) and disease-free (j) Kaplan-Meier curves with univariate analyses for patients with low versus high ELOVL2 mRNA expression in a cohort of 476 tumors. $\mathbf{k}, \mathbf{I}, \mathbf{m}, \mathbf{n}$ and $\mathbf{o}$ High-level ELOVL2 mRNA expression in neuroblastomas correlates with favorable clinical features, including low INSS stage (k), single-copy MYCN status (I), $\leq 18$ months age at diagnosis (m), low risk of PAM classification (n) and favorable Shimada/INPC (o) in a cohort of 476 tumors. ${ }^{*} P<0.05 ;{ }^{* *} P<0.01$

that enforced ELOVL2 expression diminished the induction effect of MYCN on cell proliferation (Fig. 5b) and cell cycle acceleration (Fig. 5d). Mouse xenograft results showed that enforced ELOVL2 expression diminished the promotion effect of MYCN on tumor growth (Fig. $5 \mathrm{~h}$ and i), mass (Additional file 6: Figure S6D) and re-raised the DHA content in tumors (Fig. 5j). These results demonstrated that
ELOVL2 is repressed, at least partly, for the regulation of cell proliferation by MYCN. By IHC analysis of mouse xenografts, we found that enforced MYCN expression downregulated the expression of ELOVL2 and upregulated the H2AK119ub level. In contrast, MYCN depletion upregulated the expression of ELOVL2 and downregulated the H2AK119ub level (Figs. 4k-o). 


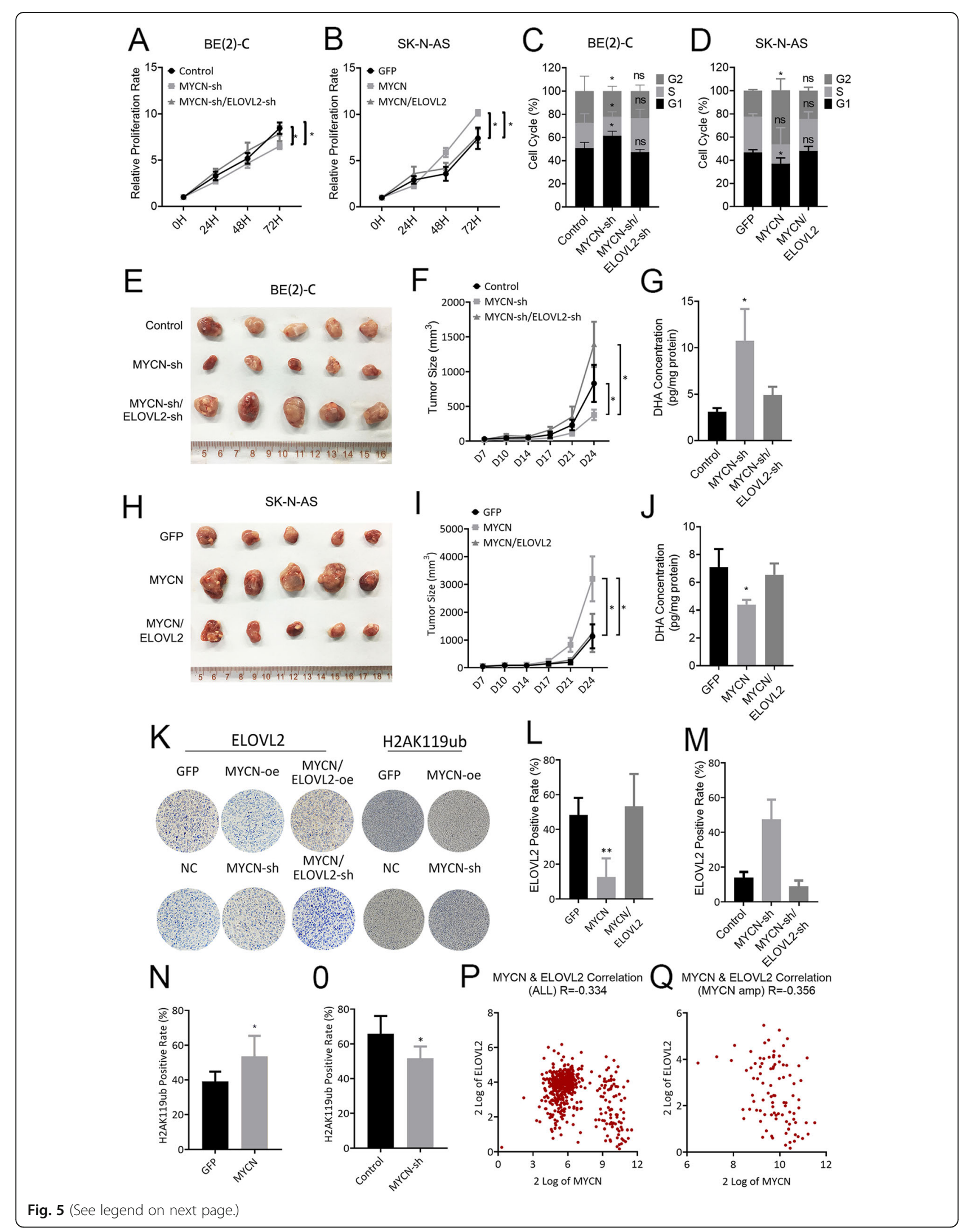




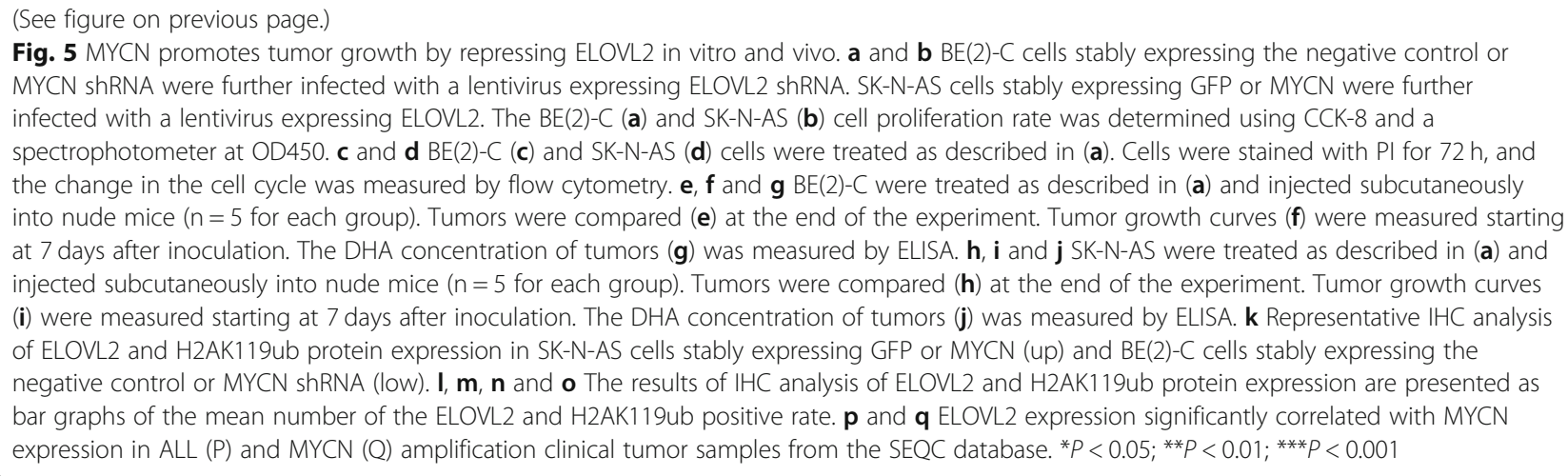

The expression level of ELOVL2 in neuroblastomas from the 496-tumor cohort was significantly correlated with MYCN status. In overall and MYCN-amplified neuroblastoma, ELOVL2 expression is significantly correlated with MYCN expression with a -0.334 and $0.356 \mathrm{R}$ value, respectively (Fig. $5 \mathrm{p}$ and $\mathrm{q}$ ).

\section{Discussion}

Unlike the vague role of $\mathrm{MYCN}$ in FA metabolism, MYC was thought to play a key role in accelerating FA and cholesterol metabolism for sustainable tumor cell growth. MYC activates the expression of the enzymes ATP citrate lyase (ACLY), acetyl-CoA carboxylase alpha (ACACA), fatty acid synthase (FASN), and stearoyl-CoA desaturase (SCD), which are all involved in fatty acid synthesis from citrate [45]. In human tumor samples, a high level of MYC in prostate cancer specifically leads to enhanced lipid metabolism [46]. In triple-negative breast cancer, MYC overexpression correlates with an increased dependency on mitochondrial FA oxidation [3]. Currently, no researchers have reported the relationship between MYCN and FA metabolism. Hence, it remains to be elucidated whether the potential regulation of FA metabolism by MYCN in neuroblastoma is different from the MYC regulation in other tumor types.

Here, we identified DHA as the strongest upregulated FA after MYCN depletion and validated the tumor suppressive properties of DHA in highly malignant MYCNamplified neuroblastoma cells. Increasing evidence has indicated the direct anti-tumor prosperity of DHA on various types of cancer cells, including neuroblastoma cells, in vitro. The mechanisms involved in the anticancer property of DHA are thought to proceed via their anti-inflammatory effects [47], their effects on the COX and LOX enzymes $[48,49]$, their effects on many transcription factors $[50,51]$, and their participation in various pathways, including activation of the Hippo pathway [52], apoptosis pathway [53] and autophagy pathway [54]. However, the regulation of DHA metabolism in tumor cells has rarely been discussed. Interestingly, mice fed a polyunsaturated fat diet enriched in DHA develop more preneoplastic foci in liver, indicating a tumorspecific DHA metabolism pattern in distinct tumor types [55]. Hence, further research on DHA metabolism is a prerequisite to fully exploiting the therapeutic application of DHA. We further unravelled that the DHA synesis enzyme ELOVL2 was remarkably upregulated after MYCN depletion and its tumor suppressive properties in this childhood malignancy both in vitro and in vivo. ELOVL2 encodes a transmembrane protein that controls the elongation of PUFA [56]. Studies have shown that ELOVL2 primarily controls the elongation process of PUFAs with 22 carbons to produce 24-carbon precursors for DHA (C22:6n-3) and DPA (C22:5n-6) formation $[57,58]$. Further research in mice indicated that Elovl2 ablation led to more impairment of systemic DHA than DPA [57]. In the literature, the function of ELOVL2 in tumor cells remains unclear and controversial. For example, in hepatocellular carcinoma, a high level of ELOVL2 was significantly associated with poor hepatocellular carcinoma (HCC) prognosis [59]. In prostate cancer, ELOVL2 showed a notable upregulation in SPOP mutations that mediate drug resistance [60]. For patients with breast cancer, ELOVL2 can be hormonally upregulated by the estrogen receptor alpha $(E R \alpha)$, and this upregulation contributes to tumor development [61]. However, a single nucleotide polymorphism (SNP) at ELOVL2 rs3734398 was significantly associated with good prognosis in cutaneous melanoma [62]. Moreover, DNA methylation at ELOVL2 is statistically significantly associated with high-risk future breast and male colorectal cancer development [63]. ELOVL2 also participates in innate and adaptive immune regulation, which might weaken the effects of immunotherapies. For example, a low level of ELOVL2 is important for maintaining $\beta$-cell function [64]. Deletion of ELOVL2 in a mouse model leads to an increase in Th1 and Th17 $\mathrm{T}$ cells and a decrease in Foxp3+ regulatory $\mathrm{T}$ cells [65]. In our experiments, the data on ELOVL2 presented here identify that declined DHA synesis caused by MYCN-mediated 
ELOVL2 inhibition in neuroblastoma cells contributes to tumor aggressiveness.

The tumor suppressive function of ELOVL2 in preclinical neuroblastoma models prompted us to decipher the transcriptional regulation of ELOVL2. We conducted IP-MS using an MYCN antibody and found that polycomb repressive complex 1 (PRC1) physically cooperated with MYCN. PRC1 depletion caused an increase in ELOVL2 expression. PRC1 acts through histone $\mathrm{H} 2 \mathrm{~A}$ monoubiquitination at lysine 119, which is comprised of three main dominant RING finger E3 ligases: BMI1, RING1B and RING1A. BMI1 was originally identified as an oncogenic cooperation factor of MYC in murine lymphomagenesis [66]. Subsequent studies have further supported an oncogenic role for BMI1 in diverse human malignancies and associated BMI1 with tumor initiation and propagation, disease progression and poor prognosis $[67,68]$. BMI1 is highly expressed in human neuroblastomas and neuroblastoma cell lines [69, 70].
Moreover, BMI1 was shown to cooperate with MYCN in the transformation of benign S-type neuroblastoma cells [71]. RING1B is highly expressed in a number of human malignancies, including prostate cancer, pancreatic ductal adenocarcinoma, ovarian cancer and urothelial bladder carcinoma [72-75]. Overexpression of RING1B has been associated with a poor prognosis for women with ovarian cancer and decreased survival times for patients with urothelial bladder carcinoma [74, 76]. Although there was no direct evidence showing that RING1B physically cooperates with MYCN, one study indicated that RING1B associates with other repressive complexes, including E2F-6.com1 , which is involved in the silencing of E2F and MYCresponsive genes in quiescent cells [77]. From our data, this new regulatory mechanism is promising because its upstream regulatory control involving $\mathrm{MYCN}$ and PRC1 is able to be disrupted with drugs reducing $\mathrm{MYCN}$ protein expression, possibly in combination with small molecular

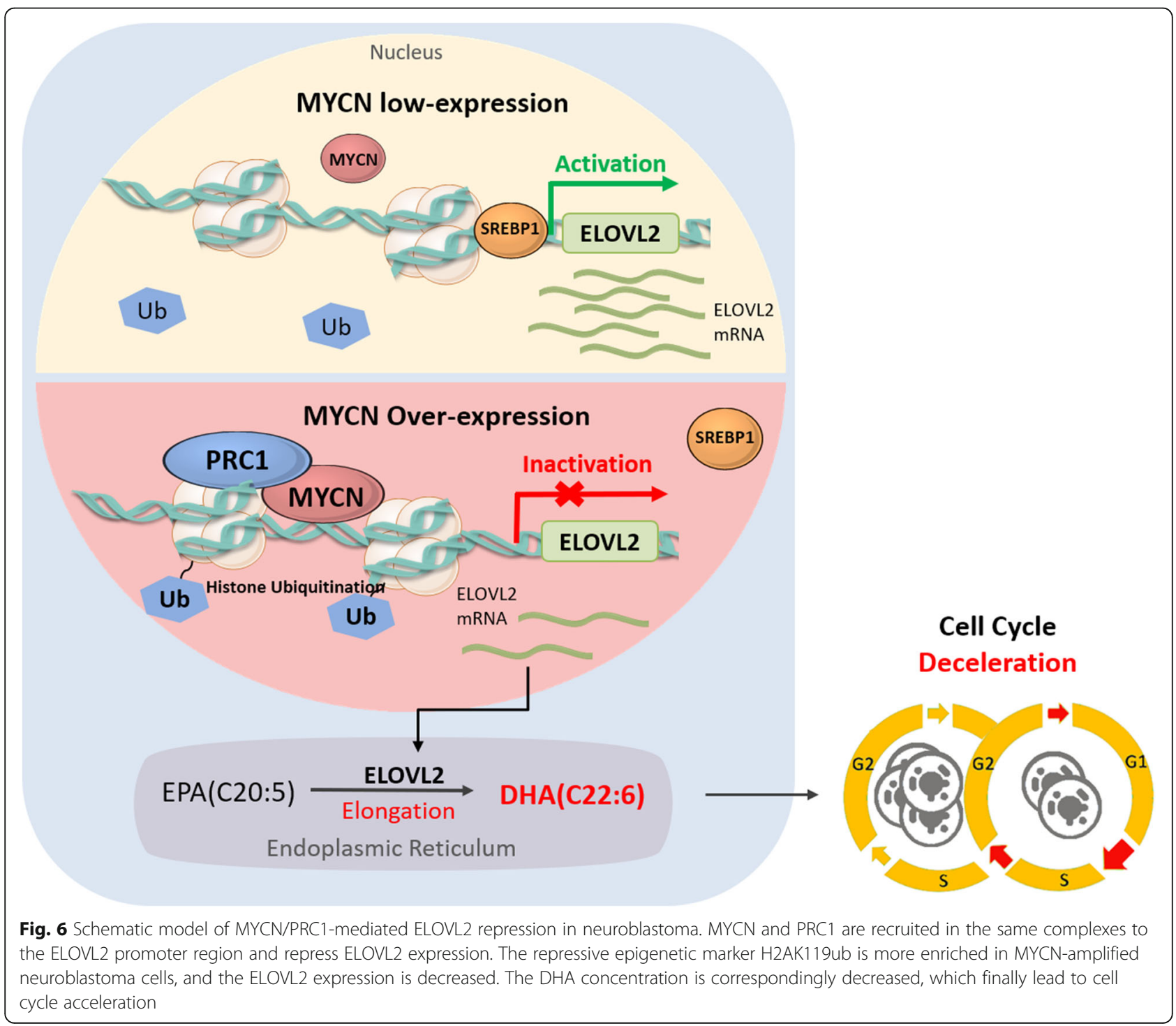


PRC1 inhibitors, such as PRT4165 [78] and PTC-209 [79]. Our studies also verified that SREBP1 is a transcriptional activator of ELOVL2 in neuroblastoma cells [43]. Interestingly, SREBP1 is an important regulator of lipogenesis and is thought to support tumor growth, such as breast tumors, renal cell carcinoma and glioblastoma [80-82]. However, clinical RNA sequencing data showed that high levels of SREBP1 expression in primary neuroblastomas correlate with clinical and molecular characteristics of a favorable tumor biology and indicates a favorable patient prognosis (data not shown). These findings suggest that SREBP1 can act as an oncogene or a tumor suppressor, depending on the tumor type.

\section{Conclusions}

In conclusion, here, we report that DHA was the strongest FA responding to treatment with MYCN depletion. In primary neuroblastomas, high expression levels of ELOVL2, which encodes DHA synesis enzyme, correlated with a established favorable clinical and molecular characteristics of tumor biology and indicates a favorable patient survival. ELOVL2 inhibited cell proliferation and the colony formation of $\mathrm{MYCN}$-amplified neuroblastoma cell lines and mice xenografts, demonstrating that ELOVL2 suppresses critical malignancy processes. ELOVL2 also responded to treatment with PRC1 siRNA, indicating the reversion of MYCN/PRC1-mediated transcriptional inhibition of ELOVL2 (modelled in Fig. 6). These data further our understanding of MYCN and PRC1 function in fatty acid metabolism, which also provides a new strategy for therapeutic intervention of highrisk MYCN-amplified neuroblastoma by jointly blocking MYCN and PRC1 activity.

\section{Supplementary information}

Supplementary information accompanies this paper at https://doi.org/10. 1186/s13046-019-1492-5.

Additional file 1: Figure S1. DHA inhibited neuroblastoma cell growth cell cycle, and tumor growth in soft agar.

Additional file 2: Figure S2. RNA-seq results from one cohort of normal brain and four cohort of neuroblastoma.

Additional file 3: Figure S3. The ELOVL2 overexpression and RNAi efficiency.

Additional file 4: Figure S4. The influence of re-supplementation with different kinds of PUFA on cell proliferation after ELOVL2 depletion.

Additional file 5: Figure S5. SREBP1 up-regulated DHA content via ELOVL2.

Additional file 6: Figure S6. Mouse xenograft tumor weight.

Additional file 7: Table S1. Patient information of tissue chip.

\section{Abbreviations}

DHA: Docosahexaenoic acid; ELOVL2: ELOVL fatty acid elongase 2; H2AK1 19ub: Histone 2A lysine 119 monoubiquitination; MYCN: MYCN ProtoOncogene, BHLH Transcription Factor; PRC1: Polycomb repressive complex 1

\section{Acknowledgements}

Not applicable.

\section{Authors' contributions}

YD: Conceptualization, Investigation, Formal Analysis, Data Curation, Writing - Original Draft, JY: Methodology, Resources, YM: Validation, Resources, TY; Visualization, XC: Validation, SG: Supervision, Writing - Review \& Editing, LW: Conceptualization, Formal Analysis, Writing - Review \& Editing, XF:

Conceptualization, Supervision, Project Administration, Funding Acquisition. All authors read and approved the final manuscript.

\section{Funding}

This study was supported by National Key R\&D Program of China (2018YFC1106100), General Financial Grant from the China Postdoctoral Science Foundation (2017M621494), the National Natural Science Foundation of China (No. 81772875, 81902804, U1932135) and The Science and Technology Commission of Shanghai (17DZ2260100, 17QA1402000, 19J(1410200)

\section{Availability of data and materials}

The datasets used and analyzed during the current study are available from the corresponding authors on reasonable request.

\section{Ethics approval and consent to participate}

All procedures performed in studies involving animals were in accordance with the ethical standards of the Ethics Committee of the Institutional Ethical Review Board of Shanghai Ninth People's Hospital.

Consent for publication

Not applicable.

\section{Competing interests}

The authors declare that they have no competing interests.

\section{Author details}

'Department of Ophthalmology, Ninth People's Hospital, Shanghai JiaoTong University School of Medicine, Shanghai 200011, China. ${ }^{2}$ Shanghai Key Laboratory of Orbital Diseases and Ocular Oncology, Shanghai 200011, China.

Received: 13 October 2019 Accepted: 25 November 2019

Published online: 19 December 2019

\section{References}

1. Beroukhim $\mathrm{R}$, et al. The landscape of somatic copy-number alteration across human cancers. Nature. 2010;463(7283):899-905.

2. Nesbit CE, Tersak JM, Prochownik EV. MYC oncogenes and human neoplastic disease. Oncogene. 1999;18(19):3004-16.

3. Dejure FR, Eilers M. MYC and tumor metabolism: chicken and egg. EMBO J. 2017;36(23):3409-20

4. Kim JW, et al. Evaluation of myc E-box phylogenetic footprints in glycolytic genes by chromatin immunoprecipitation assays. Mol Cell Biol. 2004;24(13): 5923-36.

5. David CJ, et al. HnRNP proteins controlled by c-Myc deregulate pyruvate kinase mRNA splicing in cancer. Nature. 2010;463(7279):364-8.

6. Xiang $Y$, et al. Targeted inhibition of tumor-specific glutaminase diminishes cell-autonomous tumorigenesis. J Clin Invest. 2015;125(6):2293-306.

7. Shroff EH, et al. MYC oncogene overexpression drives renal cell carcinoma in a mouse model through glutamine metabolism. Proc Natl Acad Sci U S A. 2015:112(21):6539-44.

8. Edmunds $L R$, et al. C-Myc programs fatty acid metabolism and dictates acetyl-CoA abundance and fate. J Biol Chem. 2015;290(33):20100.

9. Morrish F, et al. Myc-dependent mitochondrial generation of acetyl-CoA contributes to fatty acid biosynthesis and histone acetylation during cell cycle entry. J Biol Chem. 2010;285(47):36267-74.

10. Carter DR, et al. Glutathione biosynthesis is upregulated at the initiation of MYCN-driven neuroblastoma tumorigenesis. Mol Oncol. 2016;10(6):866-78.

11. Korshunov A, et al. Biological and clinical heterogeneity of MYCN-amplified medulloblastoma. Acta Neuropathol. 2012;123(4):515-27.

12. Ren P, et al. ATF4 and N-Myc coordinate glutamine metabolism in MYCNamplified neuroblastoma cells through ASCT2 activation. J Pathol. 2015; 235(1):90-100 
13. Ruiz-Perez MV, Henley AB, Arsenian-Henriksson M. The MYCN Protein in Health and Disease. Genes (Basel). 2017;8(4):113.

14. Zirath $\mathrm{H}$, et al. MYC inhibition induces metabolic changes leading to accumulation of lipid droplets in tumor cells. Proc Natl Acad Sci U S A. 2013;110(25):10258-63.

15. Hagenbuchner J, et al. BIRC5/Survivin enhances aerobic glycolysis and drug resistance by altered regulation of the mitochondrial fusion/fission machinery. Oncogene. 2013;32(40):4748-57.

16. Smith DJ, et al. Lack of correlation between MYCN expression and the Warburg effect in neuroblastoma cell lines. BMC Cancer. 2008;8:259.

17. Seeger RC, et al. Association of multiple copies of the N-myc oncogene with rapid progression of neuroblastomas. N Engl J Med. 1985;313(18): 1111-6.

18. Weiss WA, et al. Targeted expression of MYCN causes neuroblastoma in transgenic mice. EMBO J. 1997;16(11):2985-95.

19. Matthay KK, George RE, Yu AL. Promising therapeutic targets in neuroblastoma. Clin Cancer Res. 2012;18(10):2740-53.

20. Currie E, et al. Cellular fatty acid metabolism and cancer. Cell Metab. 2013; 18(2):153-61.

21. Bazinet RP, Laye S. Polyunsaturated fatty acids and their metabolites in brain function and disease. Nat Rev Neurosci. 2014;15(12):771-85.

22. Kim HY, et al. Inhibition of neuronal apoptosis by docosahexaenoic acid (22: 6n-3). Role of phosphatidylserine in antiapoptotic effect. J Biol Chem. 2000; 275(45):35215-23.

23. Calderon F, Kim HY. Docosahexaenoic acid promotes neurite growth in hippocampal neurons. J Neurochem. 2004;90(4):979-88.

24. Garcia MC, et al. Effect of docosahexaenoic acid on the synthesis of phosphatidylserine in rat brain in microsomes and C6 glioma cells. J Neurochem. 1998;70(1):24-30.

25. Kim HY, Spector AA. Synaptamide, endocannabinoid-like derivative of docosahexaenoic acid with cannabinoid-independent function. Prostaglandins Leukot Essent Fatty Acids. 2013;88(1):121-5.

26. Kim HY, Spector AA, Xiong ZM. A synaptogenic amide Ndocosahexaenoylethanolamide promotes hippocampal development. Prostaglandins Other Lipid Mediat. 2011;96(1-4):114-20.

27. Rashid MA, et al. N-Docosahexaenoylethanolamine is a potent neurogenic factor for neural stem cell differentiation. J Neurochem. 2013;125(6):869-84.

28. Song $\mathrm{M}$, et al. Marine omega-3 polyunsaturated fatty acid intake and risk of colorectal Cancer characterized by tumor-infiltrating T cells. JAMA Oncol. 2016;2(9):1197-206.

29. Eltweri AM, et al. Effects of Omegaven(R), EPA, DHA and oxaliplatin on oesophageal adenocarcinoma cell lines growth, cytokine and cell signal biomarkers expression. Lipids Health Dis. 2018;17(1):19.

30. Kim S, et al. omega3-polyunsaturated fatty acids induce cell death through apoptosis and autophagy in glioblastoma cells: in vitro and in vivo. Oncol Rep. 2018;39(1):239-46

31. Tan $\mathrm{RH}$, et al. Algal oil rich in $\mathrm{n}-3$ polyunsaturated fatty acids suppresses B16F10 melanoma lung metastasis by autophagy induction. Food Funct. 2018;9(12):6179-86.

32. Zarate $\mathrm{R}$, et al. Significance of long chain polyunsaturated fatty acids in human health. Clin Transl Med. 2017;6(1):25.

33. Scotting PJ, Walker DA, Perilongo G. Childhood solid tumours: a developmental disorder. Nat Rev Cancer. 2005;5(6):481-8.

34. Brodeur GM. Neuroblastoma: biological insights into a clinical enigma. Nat Rev Cancer. 2003:3(3):203-16

35. Grimmer MR, Weiss WA. Childhood tumors of the nervous system as disorders of normal development. Curr Opin Pediatr. 2006;18(6):634-8.

36. Cotterman R, Knoepfler PS. N-Myc regulates expression of pluripotency genes in neuroblastoma including lif, klf2, klf4, and lin28b. PLoS One. 2009; 4(6):e5799.

37. Harel T, et al. Homozygous mutation in MFSD2A, encoding a lysolipid transporter for docosahexanoic acid, is associated with microcephaly and hypomyelination. Neurogenetics. 2018;19(4):227-35.

38. Kerosuo $L$, et al. Enhanced expression of MycN/CIP2A drives neural crest toward a neural stem cell-like fate: implications for priming of neuroblastoma. Proc Natl Acad Sci U S A. 2018;115(31):E7351-60.

39. Yarbrough ML, et al. Frequency of Instrument, Environment, and Laboratory Technologist Contamination during Routine Diagnostic Testing of Infectious Specimens. J Clin Microbiol. 2018;56(6):e00225-18.

40. Zhao Y, et al. A high-throughput SNP discovery strategy for RNA-seq data. BMC Genomics. 2019;20(1):160.
41. Chai P, et al. Dynamic chromosomal tuning of a novel GAU1 Incing driver at chr12p13.32 accelerates tumorigenesis. Nucleic Acids Res. 2018;46(12): 6041-56.

42. Polavarapu S, et al. Effect of polyunsaturated fatty acids and their metabolites on bleomycin-induced cytotoxic action on human neuroblastoma cells in vitro. PLoS One. 2014;9(12):e114766.

43. Oishi Y, et al. SREBP1 contributes to resolution of pro-inflammatory TLR4 signaling by reprogramming fatty acid metabolism. Cell Metab. 2017;25(2): $412-27$.

44. Zhang W, et al. Comparison of RNA-seq and microarray-based models for clinical endpoint prediction. Genome Biol. 2015;16:133.

45. Stine ZE, et al. MYC, metabolism, and Cancer. Cancer Discov. 2015;5(10): 1024-39.

46. Priolo C, et al. AKT1 and MYC induce distinctive metabolic fingerprints in human prostate cancer. Cancer Res. 2014;74(24):7198-204.

47. Merendino $\mathrm{N}$, et al. Dietary omega -3 polyunsaturated fatty acid DHA: a potential adjuvant in the treatment of cancer. Biomed Res Int. 2013;2013: 310186

48. Serini $\mathrm{S}$, et al. DHA induces apoptosis and differentiation in human melanoma cells in vitro: involvement of HuR-mediated COX-2 mRNA stabilization and beta-catenin nuclear translocation. Carcinogenesis. 2012; 33(1):164-73.

49. Ho CF, et al. Expression of DHA-metabolizing enzyme Alox15 is regulated by selective histone acetylation in neuroblastoma cells. Neurochem Res. 2018;43(3):540-55.

50. Sun $\mathrm{H}$, et al. Peroxisome proliferator-activated receptor gamma-mediated up-regulation of syndecan-1 by $\mathrm{n}-3$ fatty acids promotes apoptosis of human breast cancer cells. Cancer Res. 2008;68(8):2912-9.

51. Sun C, Wei ZW, Li Y. DHA regulates lipogenesis and lipolysis genes in mice adipose and liver. Mol Biol Rep. 2011;38(2):731-7.

52. Wang J, et al. FFAR1-and FFAR4-dependent activation of hippo pathway mediates DHA-induced apoptosis of androgen-independent prostate cancer cells. Biochem Biophys Res Commun. 2018;506(3):590-6.

53. Abdi J, et al. Omega-3 fatty acids, EPA and DHA induce apoptosis and enhance drug sensitivity in multiple myeloma cells but not in normal peripheral mononuclear cells. J Nutr Biochem. 2014;25(12):1254-62.

54. Du J, et al. DHA inhibits proliferation and induces ferroptosis of leukemia cells through autophagy dependent degradation of ferritin. Free Radic Biol Med. 2019;131:356-69.

55. Pedersen $\mathrm{KB}$, et al. Liver tumorigenesis is promoted by a high saturated-fat diet specifically in male mice and is associated with hepatic expression of the proto-oncogene Agap2 and enrichment of the intestinal microbiome with Coprococcus. Carcinogenesis. 2019;40(2):349-59.

56. Bacalini MG, et al. Systemic age-associated DNA Hypermethylation of ELOVL2 gene: in vivo and in vitro evidences of a cell replication process. J Gerontol A Biol Sci Med Sci. 2017;72(8):1015-23.

57. Pauter AM, et al. Elovl2 ablation demonstrates that systemic DHA is endogenously produced and is essential for lipid homeostasis in mice. J Lipid Res. 2014;55(4):718-28.

58. Gregory MK, Cleland LG, James MJ. Molecular basis for differential elongation of omega-3 docosapentaenoic acid by the rat Elovl5 and Elovl2. J Lipid Res. 2013;54(10):2851-7.

59. Zekri AR, et al. Molecular prognostic profile of Egyptian HCC cases infected with hepatitis C virus. Asian Pac J Cancer Prev. 2012;13(11):5433-8.

60. Yan M, et al. Identification of SPOP related metabolic pathways in prostate cancer. Oncotarget. 2017;8(61):103032-46.

61. Gonzalez-Bengtsson A, et al. Estrogen enhances the expression of the polyunsaturated fatty acid Elongase Elovl2 via ERalpha in breast Cancer cells. PLoS One. 2016;11(10):e0164241.

62. Dai W, et al. Genetic variants in ELOVL2 and HSD17B12 predict melanomaspecific survival. Int J Cancer. 2019;145(10):2619-28.

63. Durso DF, et al. Acceleration of leukocytes' epigenetic age as an early tumor and sex-specific marker of breast and colorectal cancer. Oncotarget. 2017; 8(14):23237-45

64. Kone M, et al. LKB1 and AMPK differentially regulate pancreatic beta-cell identity. FASEB J. 2014;28(11):4972-85.

65. Chiurchiu V, et al. Proresolving lipid mediators resolvin D1, resolvin D2, and maresin 1 are critical in modulating T cell responses. Sci Transl Med. 2016; 8(353):353ra111.

66. Jacobs JJ, et al. Bmi-1 collaborates with C-Myc in tumorigenesis by inhibiting CMyc-induced apoptosis via INK4a/ARF. Genes Dev. 1999;13(20):2678-90. 
67. Marsh DJ, Dickson KA. Writing Histone Monoubiquitination in Human Malignancy-The Role of RING Finger E3 Ubiquitin Ligases. Genes (Basel). 2019;10(1):67.

68. Mao L, et al. Cyclin E1 is a common target of BMl1 and MYCN and a prognostic marker for neuroblastoma progression. Oncogene. 2012;31(33): 3785-95.

69. Ochiai $\mathrm{H}$, et al. Bmi1 is a MYCN target gene that regulates tumorigenesis through repression of KIF1Bbeta and TSLC1 in neuroblastoma. Oncogene. 2010;29(18):2681-90.

70. Kamijo T. Role of stemness-related molecules in neuroblastoma. Pediatr Res. 2012;71(4 Pt 2):511-5.

71. Cui $\mathrm{H}$, et al. Bmi-1 is essential for the tumorigenicity of neuroblastoma cells. Am J Pathol. 2007;170(4):1370-8.

72. Chen S, et al. H2AK119Ub1 and H3K27Me3 in molecular staging for survival prediction of patients with pancreatic ductal adenocarcinoma. Oncotarget. 2014;5(21):10421-33.

73. Chen $\mathrm{Y}$, et al. Reversal of cisplatin resistance by microRNA-139-5pindependent RNF2 downregulation and MAPK inhibition in ovarian cancer. Am J Physiol Cell Physiol. 2018;315(2):C225-35.

74. Li XD, et al. Overexpression of RNF2 is an independent predictor of outcome in patients with Urothelial carcinoma of the bladder undergoing radical cystectomy. Sci Rep. 2016;6:20894.

75. Wei M, et al. Knockdown of RNF2 induces cell cycle arrest and apoptosis in prostate cancer cells through the upregulation of TXNIP. Oncotarget. 2017: 8(3):5323-38.

76. Su WJ, et al. RNF2/Ring1b negatively regulates $p 53$ expression in selective cancer cell types to promote tumor development. Proc Natl Acad Sci U S A. 2013;110(5):1720-5.

77. Ghosh, K., et al.. 4-Nonylphenol-enhanced EZH2 and RNF2 expression, H3K27me3 and H2AK119ub1 marks resulting in silencing of p21(CDKN1A) in vitro. Epigenomics, 2019

78. Ismail IH, et al. A small molecule inhibitor of polycomb repressive complex 1 inhibits ubiquitin signaling at DNA double-strand breaks. J Biol Chem. 2013;288(37):26944-54.

79. Dey A, et al. Inhibition of BMl1 induces autophagy-mediated necroptosis. Autophagy. 2016;12(4):659-70.

80. Perone Y, et al. SREBP1 drives Keratin-80-dependent cytoskeletal changes and invasive behavior in endocrine-resistant ERalpha breast cancer. Nat Commun. 2019;10(1):2115.

81. Yang H, et al. SREBP1-driven lipid desaturation supports clear cell renal cell carcinoma growth through regulation of NF-kappaB signaling. Biochem Biophys Res Commun. 2018;495(1):1383-8.

82. Zhu J, et al. Expression of R132H mutational IDH1 in human U87 glioblastoma cells affects the SREBP1a pathway and induces cellular proliferation. J Mol Neurosci. 2013;50(1):165-71.

\section{Publisher's Note}

Springer Nature remains neutral with regard to jurisdictional claims in published maps and institutional affiliations.

Ready to submit your research? Choose BMC and benefit from:

- fast, convenient online submission

- thorough peer review by experienced researchers in your field

- rapid publication on acceptance

- support for research data, including large and complex data types

- gold Open Access which fosters wider collaboration and increased citations

- maximum visibility for your research: over $100 \mathrm{M}$ website views per year

At BMC, research is always in progress.

Learn more biomedcentral.com/submissions 